\title{
Intracorneal migration of a silicone strap
}

\author{
J. L. PEARGE AND M. J. ROPER-HALL \\ Birmingham
}

Encircling operations used in the treatment of retinal detachment have been carried out for many years and several cases have been reported of erosion of a suture through the sclera into the vitreous. Smith and Zimmerman (1965) described intraocular erosion of a polyethylene tube with a silicone plate. Kurz and Ezrow (1965) described the erosion of an encircling silicone band through the sclera into the choroid. Pischel (1965) described the anterior migration of a silicone rod or band so that it became obvious under the conjunctiva, anterior to the muscle insertions. Redi (1964) reported anterior migration of a Supramid suture into the cornea. Stanworth and Sethi (1966) described the 5 to $6 \mathrm{~mm}$. anterior migration of a silicone band from its original equatorial bed, so that it was seen ophthalmoscopically as a marked ridge pushing the ciliary body and the choroid inwards.

The case here reported is of interest in that anterior migration of a silicone band occurred some 12 months after a still successful operation for retinal detachment. The final position of the band was intracorneal.

\section{Case report}

A 68-year-old myopic woman with rheumatoid arthritis, hypertension, and obesity had an uncomplicated right intracapsular lens extraction on November 18, 1963, followed on November 25, 1963, by an uncomplicated left intracapsular lens extraction (M.J.R-H.). Postoperatively the corrected visual acuity was $6 / 9$ in each eye.

MARCH, I 965

The visual acuity in the right eye remained 6/9 with correction, but that in the left had deteriorated to hand movements. She was found to have an inferior retinal detachment of the left eye with macular involvement, and two equatorial breaks in the lower temporal quadrant.

MARGH 22, 1965

An encircling silicone strap was placed around the equator of the left eye underneath the rectus muscles, and was secured by non-absorbable scleral sutures in each quadrant. The subretinal fluid was drained by diathermy punctures (M.J.R-H.). Postoperatively the retina was found to be flat with a good ridge from the silicone strap. The visual acuity improved to $6 / 36$ with correction.

MAY, 1965

The left eye became painful and congested and the patient was taken to another hospital because of difficulty in transportation. There she was found to have a corneal haze, fine keratic precipitates, a flare and cells in the anterior chamber, and a hypopyon. The visual acuity was recorded as inaccurate perception of light. The vitreous was hazy, obscuring the fundus. A conjunctival culture was negative and a vitreous abscess was diagnosed. Treatment was given with mydriatics and subconjunctival and systemic steroids. The hypopyon gradually absorbed and the vitreous became clearer. 
JUNE, I 965

When the patient was discharged the visual acuity in the left eye was recorded as counting fingers at. 2 feet. The vitreous had become clear and it was recorded that there was a "lesion in the anterio $\overrightarrow{\vec{F}^{\prime}}$ chamber".

JULY, I 965

An ophthalmologist noted that the left eye was quiet but that conjunctiva was extending over the्ठ cornea nasally, and it was suggested that there might have been an early ciliary staphyloma. Subo sequently, the patient was unable to attend as an out-patient because of deterioration in her generaL condition.

JANUARY, I 966

She was seen by us at her home, as the left eye had again become painful and red.

Examination

The visual acuity in the left eye was $6 / 36$ with glasses. There was a mild anterior uveitis and it was found that the silicone strap had migrated forwards, encroaching beyond the limbus into the corneao between the 7 and I I o'clock meridians (Figs I and 2).

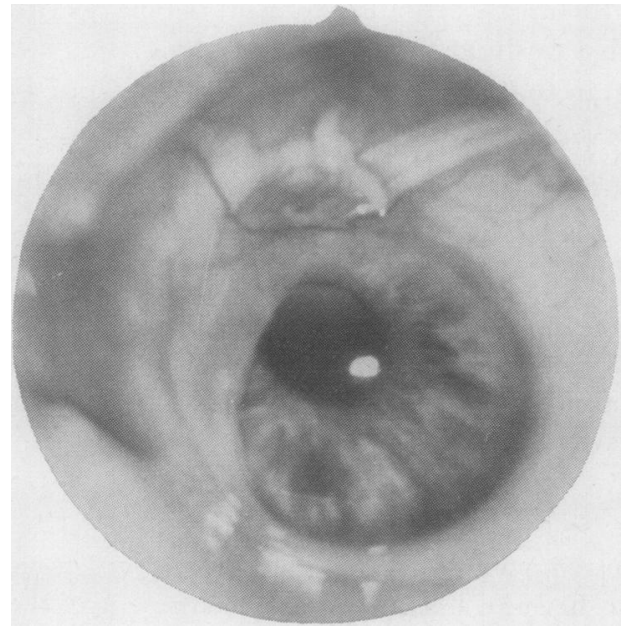

FIG. I Preoperative appearance, showing intracorneal position of silicone band with scleral suture, above, lying free on the conjunctiva

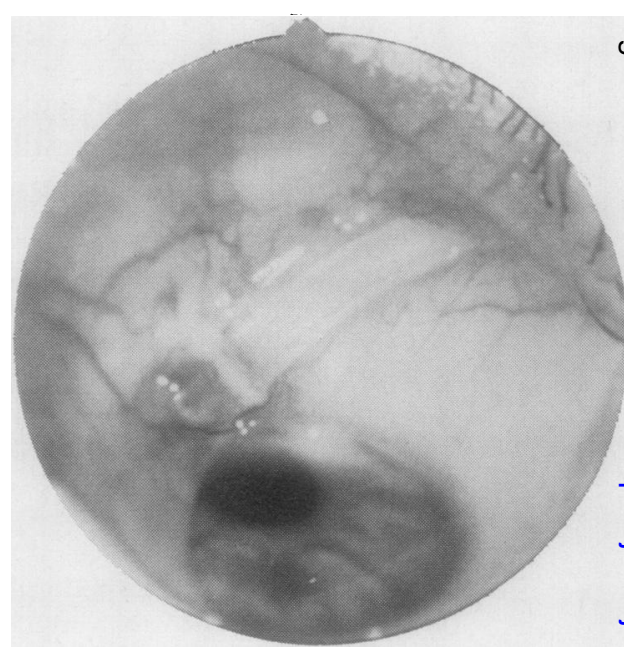

FIG. 2 Preoperative appearance, showing severaB millimetres of free silicone band on the conjunctiva

The strap was exposed on the upper part of the sclera at 12 o'clock, from which point it returneo subconjunctivally to pass behind the insertion of the temporal and inferior rectus muscles. One suture was seen around the strap at its exit from the corneal tunnel above. Apart from some restriction of elevation, the external ocular movements were full. Examination of the structuresu of the anterior chamber with a Koeppe contact lens showed that the strap was lodged within the cornea and was projecting as a ridge into the anterior chamber (Fig. 3, opposite). Ophthalmo응 scopically there was a good ridge in the temporal fundus and the retina was in place.

FEBRUARY, I 966

Operation (M.J.R-H. and J.L.P.)

Under local anaesthesia the conjunctiva was incised in the lower temporal quadrant. The joined $\frac{\overrightarrow{\mathbb{P}}}{\mathbb{Z}}$ ends of the silicone strap were located and the retaining sutures cut. The strap was also cut where itor 


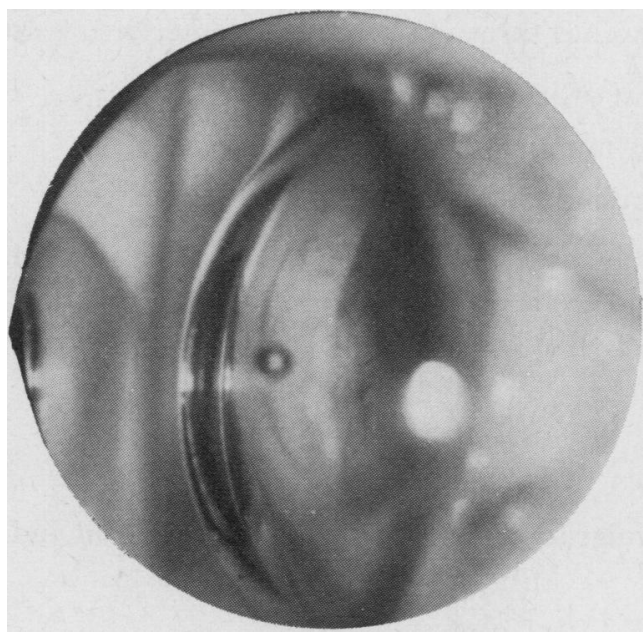

FI G. 3 Preoperative gonioscopic appearance, showing intracorneal tunnel projecting into the anterior chamber

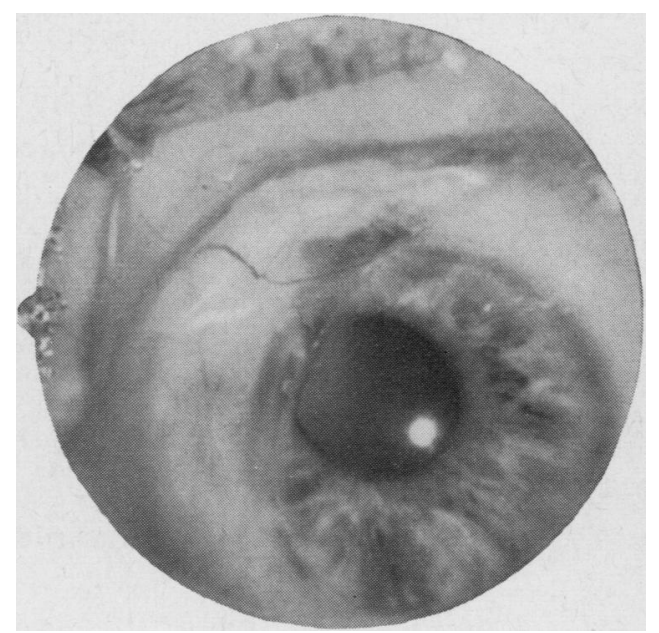

FIG. 4 Immediate postoperative appearance after removal of silicone band

was lying free on the conjunctiva above, and it was eased out of its bed and the tunnel within the cornea. After its removal the conjunctiva was sutured and the patient made an uneventful recovery (Fig. 4). Subsequently, this eye has remained quiet with a corrected visual acuity of $6 / 36$. The intraocular pressure remains normal and the retina is flat. The peripheral visual field remains full.

\section{Discussion}

Several interesting points arise from this case.

(a) The silicone strap in its anterior migration of more than $14 \mathrm{~mm}$. must have cut through the insertion of the medial rectus and the superior rectus and, as ocular movements are now full in this eye, these muscles must have re-inserted themselves after the passage of the strap. It would appear also that the strap inferiorly had begun to erode into the insertion of the inferior rectus muscle.

(b) Originally the strap was episcleral without a buried scleral implant, yet it was finally found to be intracorneal, so that at some point in its anterior migration it had eroded into the corneo-sclera. In this patient it is possible that migration and erosion were encouraged by scleromalacia associated with her rheumatoid arthritis although there was no clinical evidence of this. The suture which was still tied around the band had cut out from its original position in the sclera in the upper nasal quadrant.

(c) When the patient was seen at another clinic in April, 1965, with the diagnosis of vitreous abscess, it may well have been that the silicone strap was pressing on the ciliary body, causing ciliary irritation. When we saw her again, the eye was relatively uncongested. By this time the strap had become intracorneal and was no longer under tension.

The case reported by Stanworth and Sethi (I 966 ) had marked ciliary congestion and at this stage anterior migration of the silicone band had occurred over the ciliary body. This eye had a buried scleral implant in addition to the strap, which may have encouraged intrascleral erosion. 
Theoretically, an episcleral strap has three obstacles to its anterior migration:

(I) The scleral sutures securing it at its original position.

(2) At the insertion of the rectus muscles.

(3) At the limbal insertion of episclera and conjunctiva.

It is, therefore, possible that the strap could erode and become intrascleral at any of these points. In our case it would seem likely that erosion commenced at the limbus to become intracorneal, but there is no conclusive evidence that intrascleral erosion did not take place before.

\section{Summary}

A case is reported of intracorneal migration of an episcleral silicone band and its subsequenţ removal.

\section{References}

kURz, G. H., and Ezrow, L. (1965) Arch. Ophthal. (Chicago), 73, 183 Pischel, D. K. (1965) In "Complications in Eye Surgery", ed. R. M. Fasanella, 2nd ed., pp 299-345. Saunders, Philadelphia REDI, F. (1964) Atti Soc. oftal. Lombarda, 19, 92 Smith, м. e., and zimmerman, L. E. (1965) Arch. Ophthal. (Chicago), 73, 618 stANWORTh, A., and SETHI, B. P. (1966) Brit. 7. Ophthal., 50, 73 I 